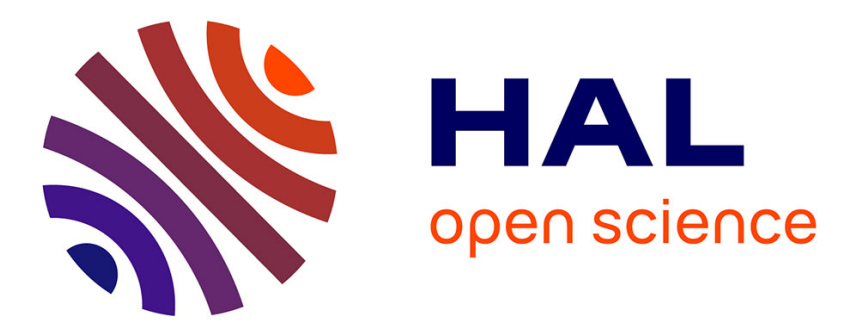

\title{
Implementation of the toroidal absorption cell with multi-layer patterns by a single ring surface
}

Hong Chang, Shiling Feng, Xuanbing Qiu, Huiyan Meng, Guqing Guo, Xiaohu He, Qiusheng He, Xiaohua Yang, Weiguang Ma, Ruifeng Kan, et al.

\section{- To cite this version:}

Hong Chang, Shiling Feng, Xuanbing Qiu, Huiyan Meng, Guqing Guo, et al.. Implementation of the toroidal absorption cell with multi-layer patterns by a single ring surface. Optics Letters, 2020, 45 (21), pp.5897-5900. 10.1364/ol.404198 . hal-03019219

\section{HAL Id: hal-03019219 \\ https://hal.univ-lille.fr/hal-03019219}

Submitted on 17 Sep 2021

HAL is a multi-disciplinary open access archive for the deposit and dissemination of scientific research documents, whether they are published or not. The documents may come from teaching and research institutions in France or abroad, or from public or private research centers.
L'archive ouverte pluridisciplinaire HAL, est destinée au dépôt et à la diffusion de documents scientifiques de niveau recherche, publiés ou non, émanant des établissements d'enseignement et de recherche français ou étrangers, des laboratoires publics ou privés. 


\title{
Implementation of the toroidal absorption cell with multi-layer patterns by a single ring surface
}

\section{HONG CHANG,${ }^{1}$ SHILING FENG,${ }^{1}$ XUANBING QIU, ${ }^{1}$ HUIYAN MENG, ${ }^{1}$ GUQING GUO, ${ }^{1}$ XIAOHU HE, ${ }^{1}$ QIUSHENG HE, ${ }^{1}$ XIAOHUA YANG, ${ }^{2}$ WEIGUANG MA, ${ }^{3}$ RUIFENG KAN, ${ }^{4}$ CHRISTA FITTSCHEN,${ }^{5}$ AND CHUANLIANG LI ${ }^{1, *}$}

\author{
${ }^{1}$ Department of Physics, Taiyuan University of Science and Technology, Taiyuan 030024, China \\ ${ }^{2}$ School of Science, Nantong University, Nantong 226019, China \\ ${ }^{3}$ State Key Laboratory of Quantum Optics and Quantum Optics Devices, Institute of Laser Spectroscopy, Shanxi University, Taiyuan \\ 030006, China \\ ${ }^{4}$ State Key Laboratory of Applied Optics, Changchun Institute of Optics, Fine Mechanics and Physics, Chinese Academy of Sciences, \\ Changchun, Jilin 130033, China \\ ${ }^{5}$ Université Lille, CNRS, UMR 8522 - PC2A - Physicochimie des Processus de Combustion et de l'Atmosphère, Lille F-59000, France \\ *Corresponding author: li_chuanliang@126.com
}

We developed a type of toroidal multi-pass cell with multi-layer patterns based on the off-axis model when combined with absorption masks. The effective path-length of the original toroidal multi-pass cell is extended several roundtrips in comparison with the single-layer pattern, since the inner surface of the toroidal multi-pass cell is more efficiently utilized. The light pattern has been achieved by using the simple ring surface that is easy to fabricate. The exact analytical equations for the design of the toroidal multi-pass cell were derived based on analytical vector calculations. A series of numerical ray tracing simulations are presented, and the maximum theoretical optical path length that can be reached is $30 \mathrm{~m}$ with a setup of $5 \mathrm{~cm}$ column radius. Furthermore, two practical spot patterns are demonstrated with a path length of $8.3 \mathrm{~m}$ for 2-layer pattern and $10 \mathrm{~m}$ for 3-layer pattern with respective volumes of $71 \mathrm{~mL}$ and $110 \mathrm{~mL}$.

Multi-pass cells (MPCs) are extensively used in detecting trace compounds diluted in gaseous and liquid media because they allow to increase the sensitivity owing to their long optical path length (OPL). Several well-known MPCs, such as Herriott cell [1-3], White cell [4] and Chernin cell [5] have been proposed and successfully used in spectroscopy to observe weak spectra of interest. Among these multi-pass cells, the design of the Herriott cell has been continuously updated and improved because it commonly consists of two opposite mirrors and is susceptible to mechanical disturbance [6]. However, 
there is only a single eclipse pattern of reflection spots and most of the mirror area is not efficiently used in the Herriott cell causing a limited optical path length. As a result, perturbers based on astigmatic or cylindrical mirrors are employed to construct the Herriott cell [7-9]. The number of light reflections in these modified cells is significantly increased and their spot distributions customarily exhibit a Lissajous-like pattern. Consequently, these cells increase their OPL and don't increase the spot overlap due to the more efficient utilization of mirror area. Recently, several novel dense-pattern Herriott cells, even for spherical mirrors, were implemented by Liu et al. and Dong et al. [10-12]. Lately, Dong et al. investigated a Herriott cell with dense spot pattern based on the spherical aberration, and they simulated a set of exotic spot patterns with highly efficient beam folding [13]. However, these cells have stringent requirements for the distance, entrance angle and tilt of the mirrors [14].

Recently, Bernacki and Tuszon et al. [15, 16] designed a compact toroidal MPC with the low-volume monolithic ring. The OPL of the toroidal MPC can be flflexibly changed by solely adjusting the entrance angle, and it is thus suitable for different absorption conditions of the sought-after species. In addition, it features mechanical robustness and lightweight, which makes it attractive for building mobile and portable laser spectroscopy. Then, Mangold and Graf et al. used respectively paraboloid and emerging segmented inner surfaces to increase the number of reflections $[17,18]$. We also proposed a spiral-torus structure cell for raising the number of refections [19]. Recently, Sun et al. [20] designed a novel structure for a circular cell based on two spherical mirrors that the heights and the position of two sphere centers are different and its maximum OPL is relevant with positions of entrance and exit holes. In practice, these spherical surfaces are easily fabricated. Simultaneously, single spherical surfaces come with the advantage of high surface quality and low cost.
Previous investigation of the toroidal cell focused on the number of reflections and numerical ray tracing modeling of single-layer [17-21]. To our knowledge, the analytical method that is of significant importance for the design of multi-layer patterns in a circular MPC has not been reported.

In this Letter, a new dense-pattern toroidal multi-pass cell with a ring surface and the absorption mask is proposed and polygon star spot patterns can be generated on multiple layers of the inner surface of the toroidal cell due to the off-axis irradiation. The design equations for exact analytical solutions to the toroidal cell are derived based on the analytical vector calculations and the astigmatism of our several designs is shown as images. Two typical ray tracing simulations of two- and three-layer patterns are presented, and two practical experimental demonstrations of the two-layer and three-layer pattern with separately $8.3 \mathrm{~m}$ and $71 \mathrm{~mL}$ and $10 \mathrm{~m}$ and $110 \mathrm{~mL}$ are demonstrated.

ABCD matrix is the simple mathematical form for ray tracing calculations at the condition of paraxial approximation. However, it can not well describe the ray propagation of a circular cell because the paraxial theory ( $\sin \theta \approx \theta$, where $\theta$ is the angle between incident ray and optical axis) may be invalid and the direction of the optical axis rotates with reflections. The ray-tracing configuration is shown in Fig. 1(a). The $M_{0}\left(x_{0}, y_{0}, z_{0}\right)$ is the ray injection position and $M_{0}{ }^{\prime}\left(x_{0}^{\prime}, y_{0}^{\prime}, 0\right)$ is the projection of $M_{0}$ in the $x-y$ plane. When the setup fulfills the stability conditions of cavity, the beam will be confined in the cell and travels back and forth. The stability condition is $L<2 R$ within the paraxial theory, and it has also been proved true in the circular cell by Sun et al. [21]. Herein, $L$ is the separation distance of two mirrors and $R$ is the radius of curvature. The off-axis incident beam propagating in the cell results in reflections on different layers by the interior surface of 
the cell. Finally, the trajectory of light forms a polygon star at each layer and the beam exits the cell from the incident hole.
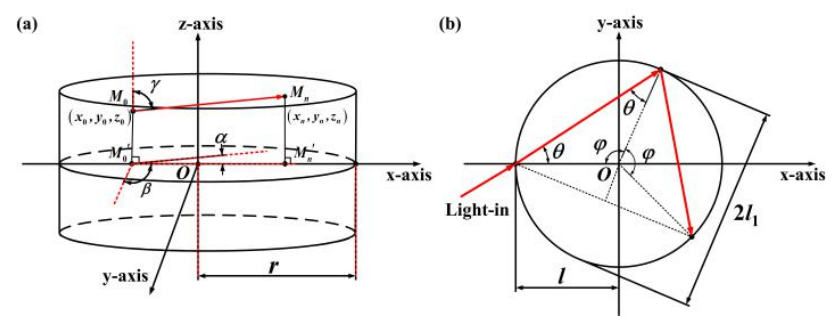

Fig. 1. (a) Propagation of the light rays from position $M_{0}$ (the incident point) to position $M_{\mathrm{n}}$ (after the $n$th pass) in the three-dimensional rectangular coordinate system. $M_{0}{ }^{\prime}$ and $M_{\mathrm{n}}{ }^{\prime}$ are the projections of the $M_{0}$ and $M_{\mathrm{n}}$ in the $x$ - $y$ plane; $\alpha, \beta$ and $\gamma$ are the three axial-twist angles between the incident ray and the three axes; $r$, tangential curvature in the $x$-y plane. (b) Top view of the reflections. $\theta$, the incident angle of the spot pattern in the $x-y$ plane; $O$, the origin of the coordinate system; $l$, the horizontal distance between the spot and the z-axis; $l_{1}$, the distance after rotation to the $\mathrm{z}$-axis in the normal direction.

The geometric structure of transmitting ray is shown in Fig. 1. Figure 1(a) and (b) are the stereogram and plan schematics. It should be notified that $r$ is the radius with respect to the middle plane $(\mathrm{z}=0)$ and $l$ corresponds to the radii of each layer for spot patterns. As shown in Fig. 1, the incident angle $\theta$ satisfies:

$$
\theta=\frac{\pi}{2} \cdot\left[1-\frac{1+x}{m}\right]
$$

Where $x$ is the number of times the beam passes the $x$-axis and $m$ is a nonzero number and is the number of spots per row. According to the Eq. $(*)$, we can derive all solutions of the $\theta$ with various values of the $x$. For the case with $m=5$, the $\theta$ has two solutions of $18^{\circ}$ and $54^{\circ}$ and the values of $x$ are 3 and 1 . In our work, we need the long optical length, hence we present the following Eq. (1) for the unique solution that can calculate the longest optical length in all solutions of the $\theta$.

$$
\theta=\left\{\begin{array}{l}
\frac{\pi}{2 m}, m=j ; \\
\frac{\pi}{m / 2}, m=2 j ; \\
\frac{\pi}{m}, \text { otherwise. }
\end{array}\right.
$$

Where we assume $j$ is an arbitrary positive odd number, such as, $1,3,5,7$ and so on. There are three cases for the values of $m$ in Eq. (1). According to Eq. (1), we know that the value of $\theta$ depends on the multiple relationships between the values of $m$ and $j$. For instance, for $m=15, \theta$ equals to $6^{\circ}$; if $m=30$, the corresponding $\theta$ is $12^{\circ}$; for $m=36$, according to the case three, as a result, the value of $\theta$ is $5^{\circ}$. For the two-layer pattern, satisfying the closed path condition, $m$ fulfills the following condition

$$
m=4 k+a,
$$

where $k \in \mathrm{N}$ and the values of $a$ are defined as $0,1,2$ and 3. The analogous relationship for three-layer spot patterns is derived as follows:

$$
m=6 k+a .
$$

Herein, the values of $a$ are defined as $0,1,2,3,4$ and 5 .

In order to trace the trajectory of rays, we construct the vectors method to calculate each spot coordinate in the 3-D Cartesian system. After $n$ passes, the corresponding values of the rays could be expressed by the following equation:

$$
d_{n}=\frac{x_{n}-x_{n-1}}{\boldsymbol{a}_{\boldsymbol{n}}}=\frac{y_{n}-y_{n-1}}{\boldsymbol{b}_{\boldsymbol{n}}}=\frac{z_{n}-z_{n-1}}{\boldsymbol{c}_{\boldsymbol{n}}} .
$$

where $\boldsymbol{a}_{\boldsymbol{n}}, \boldsymbol{b}_{\boldsymbol{n}}$ and $\boldsymbol{c}_{\boldsymbol{n}}$ are the components of the direction vector along the respective $x, y$ and $z$ axes at the $n$th pass. They are given as follows:

$$
a_{n}=k_{1 \mathrm{n}}+a_{n-1}, b_{n}=k_{2 \mathrm{n}}+b_{n-1}, c_{n}=k_{3 n}+c_{n-1} .
$$

The variables with script $n-1$ represent the parameters at the (n-1)th pass count. 
(a)

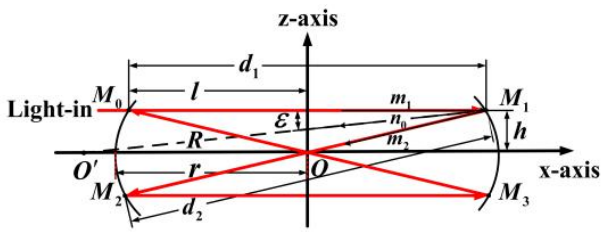

(b)

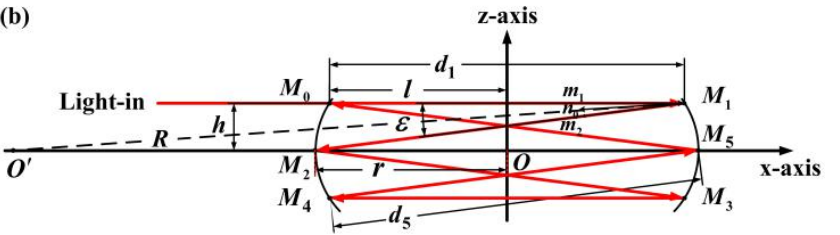

Fig. 2. Transmission of the rays in the $x-z$ plane. $d_{1,2 \ldots n}$, the path-length of the $1,2 \ldots$ th pass count on the inner surface of the toroidal cell; $r$, the radius of curvature in the tangential direction in the $x-y$ plane, which is equal to the cell radius; $h$, the vertical distance of the entrance beam in the $z$-axis and equals to $x_{0}$; $R$, the sagittal curvature of the toroidal surfaces; $O^{\prime}$, the center of the circle with the radius of $R ; \boldsymbol{m}_{1}, \boldsymbol{m}_{2}$, the vectors of the input beam and the reflected beam; $\boldsymbol{n}_{\mathbf{0}}$, the normal vector of the toroidal surface;

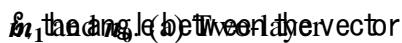
distribution of the spots on the toroidal mirror surface. (b) Three-layer distribution of the spots on the toroidal mirror surface.

Likewise, $\boldsymbol{k}_{\mathbf{1 n}}, \boldsymbol{k}_{\mathbf{2 n}}$ and $\boldsymbol{k}_{\mathbf{3 n}}$ are the vectors of the normal vector $\boldsymbol{n}_{\mathbf{0}}$ along the $x, y$ and $z$ axes, and their corresponding magnitudes $\left(k_{1 \mathrm{n}}, k_{2 \mathrm{n}}\right.$ and $\left.k_{3 \mathrm{n}}\right)$ are $k_{1 n}=-4 \cdot x_{n} \cdot \frac{2 \cdot a_{n} \cdot x_{n}+2 \cdot b_{n} \cdot y_{n}+(-2) \cdot c_{n} \cdot z_{n} \cdot \frac{\left(R-r-\sqrt{R^{2}-z_{n}{ }^{2}}\right)}{\sqrt{R^{2}-z_{n}^{2}}}}{4 \cdot x_{n}{ }^{2}+4 \cdot y_{n}{ }^{2}+\left\{(-2) \cdot z_{n} \cdot \frac{\left(R-r-\sqrt{R^{2}-z_{n}^{2}}\right)}{\sqrt{R^{2}-z_{n}^{2}}}\right\}^{2}}$,

$$
k_{2 n}=-4 \cdot y_{n} \cdot \frac{2 \cdot a_{n} \cdot x_{n}+2 \cdot b_{n} \cdot y_{n}+(-2) \cdot c_{n} \cdot z_{n} \cdot \frac{\left(R-r-\sqrt{R^{2}-z_{n}^{2}}\right)}{\sqrt{R^{2}-z_{n}^{2}}}}{4 \cdot x_{n}{ }^{2}+4 \cdot y_{n}{ }^{2}+\left\{(-2) \cdot z_{n} \cdot \frac{\left(R-r-\sqrt{R^{2}-z_{n}^{2}}\right)}{\sqrt{R^{2}-z_{n}^{2}}}\right\}^{2}},
$$$$
k_{3 n}=4 \cdot z_{n} \cdot \frac{\left(R-r \cdot \sqrt{R^{2}-z_{n}^{2}}\right)}{\sqrt{R^{2}-z_{n}^{2}}} \cdot \frac{2 \cdot a_{n} \cdot x_{n}+2 \cdot b_{n} \cdot y_{n}+(-2) \cdot c_{n} \cdot z_{n} \cdot \frac{\left(R-r-\sqrt{R^{2}-z_{n}^{2}}\right)}{\sqrt{R^{2}-z_{n}^{2}}}}{4 \cdot x_{n}{ }^{2}+4 \cdot y_{n}{ }^{2}+\left\{(-2) \cdot z_{n} \cdot \frac{\left(R-r-\sqrt{R^{2}-z_{n}^{2}}\right)}{\sqrt{R^{2}-z_{n}^{2}}}\right\}} .
$$

Where $a_{n}, b_{n}$ and $c_{n}$ are the corresponding magnitudes of $\boldsymbol{a}_{n}$, $\boldsymbol{b}_{\boldsymbol{n}}$ and $\boldsymbol{c}_{\boldsymbol{n}}$, respectively. The values of $a_{n}, b_{n}$ and $c_{n}$, and their transitive relation can be calculated using the trigonometrical function. The expression of $y_{n}$ is derived by substituting Eqs. (5) and (6) into Eq. (4).

$$
y_{n}=-\frac{2 \cdot\left\{\frac{a_{n}}{b_{n}} \cdot\left(x_{n-1}-y_{n-1} \cdot \frac{a_{n}}{b_{n}}\right)+\frac{c_{n}}{b_{n}} \cdot\left(z_{n-1}-y_{n-1} \cdot \frac{c_{n}}{b_{n}}\right)\right\}}{\left\{\left(\frac{a_{n}}{b_{n}}\right)^{2}+\left(\frac{c_{n}}{b_{n}}\right)^{2}+1\right\}}-y_{n-1} .
$$

$$
x_{n}=\left(y_{n}-y_{n-1}\right) \cdot \frac{a_{n}}{b_{n}}+x_{n-1}, z_{n}=\left(y_{n}-y_{n-1}\right) \cdot \frac{c_{n}}{b_{n}}+z_{n-1} \cdot
$$

Then, it is straightforward to obtain the values of $d_{n}, x_{n}$ and $z_{n}$ according to Eq. (4). Therefore, we can derive the Eq. (8) which is about $x_{n}$ and $z_{n}$. The desired OPL can be flexibly constructed by a pair of $m$ and $d_{n}$, by simply changing the incidence angle. In principle, the above expressions about the ray transmission can be used with any number of spot-layers without taking consideration of the astigmatism.

Four examples, as plotted in Fig. 3, show the sequent propagation paths of the beam in the toroidal cell. For clarity, the distance between spot layers in Fig. 3(a) and (b) is $5 \mathrm{~mm}$, and the number of each layer reflection is set to ten. The largest numbers of reflections for two and three-layer are 200 and 300 illustrated in Fig. 3(c) and (d), respectively. As a result of $5 \mathrm{~mm}$ layer height, the ratios of OPL to volume for the two and three-layer pattern reach 28 
$\mathrm{cm}^{-2}$ and $27 \mathrm{~cm}^{-2}$, respectively. The ratios of OPL to volume is derived by OPL divided by the effective volume of cell. According to the mathematical formula $V=\pi r^{2} h$, we calculate the effective volume of cell. For example, the OPL is $20 \mathrm{~m}$ and the effective cell volume is $71 \mathrm{~mL}$, so the corresponding ratio is $28 \mathrm{~cm}^{-2}$.
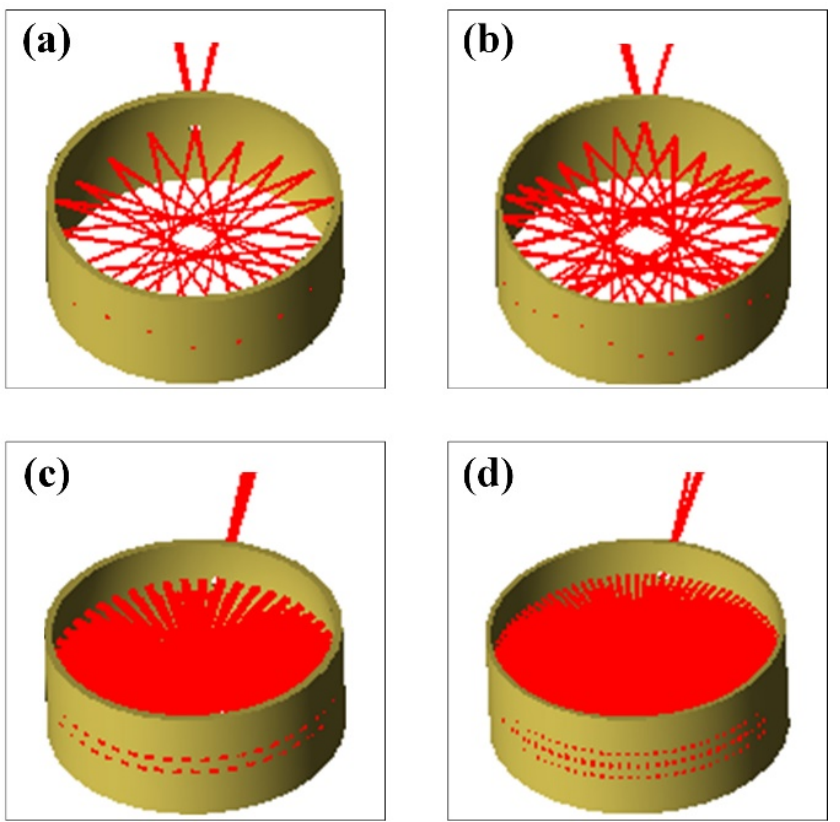

Fig. 3. (a) A two-layer example with 20 passes. (b) A three-layer example with 30 passes. (c) The largest number of reflections (200 times) among two-layer simulating cases with $r=50 \mathrm{~mm}, R=100$ mm and $71 \mathrm{~mL}$. (d) The largest number of reflections (300 times) among three-layer simulating cases with $r=50 \mathrm{~mm}, R=200 \mathrm{~mm}$ and $110 \mathrm{~mL}$. (a)
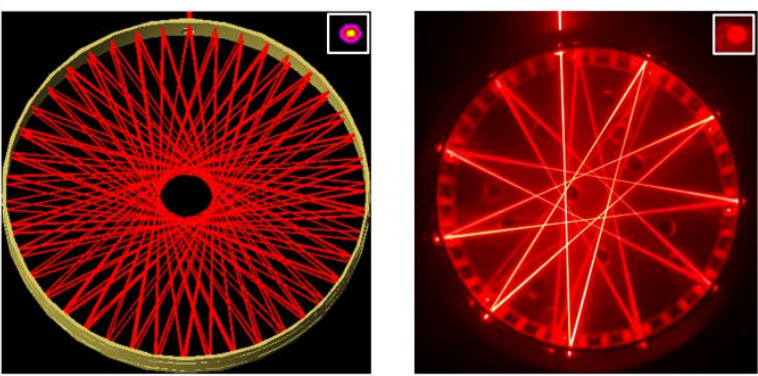

(b)
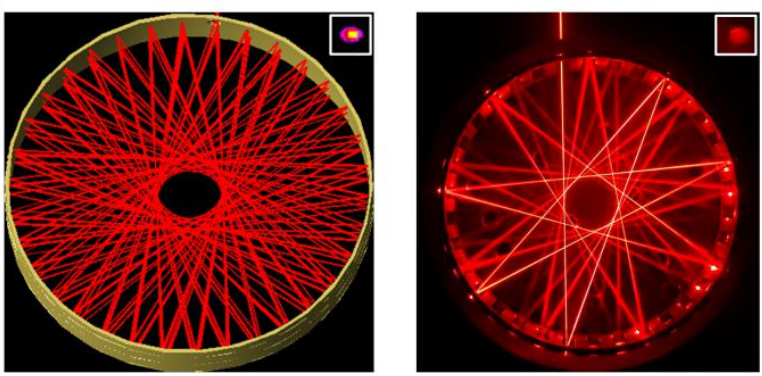

Fig. 4. Calculated (left) and practical (right) beam patterns and their light shapes of the output beam of (a) the two-layer and (b) the three-layer spot patterns. The inserts show the exit beam spot for each pattern.

The calculated and practical spot patterns are exhibited in Fig. 4. Herein, a diode laser emitting at $635 \mathrm{~nm}$ and the absorption masks were employed to observe the practical spot patterns. In the Fig. 4(a) and (b), the incident angle $\theta$ is adjusted respectively as $8.6^{\circ}$ and $10.6^{\circ}$ to achieve 84 passes and 102 passes; the effective depths of the cell are 9 and $14 \mathrm{~mm}$. Therefore, the OPLs of our practical cell are $8.3 \mathrm{~m}$ and $10 \mathrm{~m}$ and their corresponding ratios are 12 and 9 $\mathrm{cm}^{-2}$, respectively.

(a)

(b)
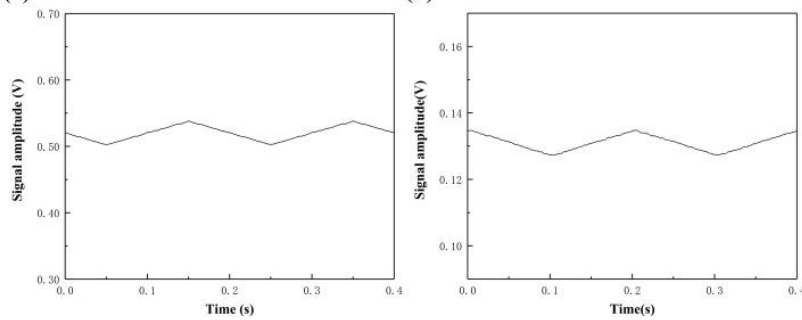
Fig. 5. The baseline measurement of the output beam of (a) the two-layer and (b) the three-layer spot patterns corresponding to the inserts of Fig. 4(a) and (b).

The off-axis reflections may result in astigmatism, but the phenomenon is not serious for the two and three-layer pattern, as depicted in the inserts of Fig. 4. Consequently, we simulated the irradiance maps of the spot emitted within the last reflection to express the astigmatism of the design. This indicates that the calculated beam pattern and position for toroidal absorption cell are consistent with the experimental. Furthermore, the quality of practical output beam profile is considerably improved when equipped with the designed mask. The experimental profiles are basically consistent with the theoretical ones, and the astigmatism is neglectable.

Moreover, in order to verify the capability of the fringe suppression of the cell, we added the absorption mask in it and used the triangular wave signal within the frequency of $5 \mathrm{~Hz}$ generated by a signal generator to scan the laser frequency. In addition, the mask has been designed and employed in the cell, and the etalon effect has been significantly suppressed in detection. For further validating the fringe suppression, the baseline measurement results are shown in Fig.5 and the fringe-noise level of the two-layer and the three-layer spot patterns are $0.35 \%$ and $0.21 \%$, respectively, comparable with the 1-layer pattern of the Ref. [22].

In summary, we reported the implementation of a multi-layer spot pattern in a toroidal multi-pass cell with a simple ring surface and absorption masks. The analytical equations for general solutions for the ray trajectory in the toroidal multi-pass cell are obtained according to the analytical vector calculations. The monolith nature of the cell and the low volume guarantee robustness, rapid response and simple adjustment. Two prototypes of practical use of the cell are demonstrated by adjusting different incident angles resulting in OPL of $8.3 \mathrm{~m}$ and 10 $\mathrm{m}$ within a volume of 71 and $110 \mathrm{~mL}$, respectively. Due to highly efficient utilization of the inner surface, the effect of optical fringes will be reduced at the benefit of higher efficient utilization of mirror area in comparison with the single layer toroidal cell. This makes this new design suitable for a variety of spectroscopic applications.

Funding. This work was supported by the National Natural Science Foundation of China (Grant Nos. U1810129, and 11904252), State Key Laboratory of Applied Optics (SKLAO-201902), Transformation of Scientific and Technological Achievements Fund of Shanxi Province (201904D131025), Excellent Youth Academic Leader in Higher Education of Shanxi Province (2018), Key Research and Development Program of Shanxi Province of China (Grant Nos. 201803D31077 \& 201803D121090), the Fund for Shanxi “1331 Project” Key Innovative Research Team (1331KIRT), Natural Science Foundation of Shanxi Province of China (No. 201801D221017) and the Fund for Shanxi Key Subjects Construction.

Disclosures: The authors declare that there are no conflicts of interest related to this article. The authors declare no conflicts of interest.

\section{References}

1. D. Herriott, H. Kogelnik, and R. Kompfner, Appl. Opt. 3, 523 (1964).

2. X. Guo, F. Zheng, C. Li, X. Yang, N. Li, S. Liu, J. Wei, X. Qiu, and Q. He,

Opt. Lasers Eng. 115, 243 (2019). 
3. C. Li, L. Shao, H. Meng, J. Wei, X. Qiu, Q. He, W. Ma, L. Deng, and Y. Chen, Opt. Express 26, 29330 (2018).

4. J. U. White, J. Opt. Soc. Am. 32, 285 (1942).

5. Y. Wang, Z. Li, X. Liu, F. Fang, and X. Zhang, Appl. Opt. 56, 8541 (2017).

6. C. Robert, Appl. Opt.46, 5408 (2007).

7. D. R. Herriott and H. J. Schulte, Appl. Opt. 4, 883 (1965).

8. J. Altmann, R. Baumgart, and C. Weitkamp, Appl. Opt. 20, 995 (1981).

9. J. Sliver, Appl. Opt. 44, 6545 (2005).

10. K. Liu, L. Wang, T. Tan, G. S. Wang, W. J. Zhang, W. D. Chen, and X. M. Gao, Sens. Actuators, B 220, 1000 (2015).

11. C. Li, L. Dong, C. Zheng, and F. K. Tittel, Sens. Actuators, B 232, 188

(2016).

12. L. Dong, C. Li, N. P. Sanchez, A. K. Gluszek, R. J. Griffin, and F. K. Tittel, Appl. Phys. Lett. 108, 011106 (2016).

13. R. Cui, L. Dong, H. Wu, S. Li, X. Yin, L. Zhang, W. Ma, W. Yin, and F. Tittel, Opt. Lett. 44, 1108 (2019).

14. A. Manninen, B. Tuzson, H. Looser, Y. Bonetti, and L. Emmenegger, Appl. Phys. B 109, 461 (2012)

15. B.. Bernacki, U.S. Patent 7876443 (25 January 2011).

16. B. Tuzson, M. Mangold, H. Looser, A. Manninen, and L. Emmenegger, Opt. Lett. 38, 257 (2013)

17. M. Mangold, B. Tuzson, M. Hundt, J. Jágerská, H. Looser, and L. Emmenegger, J. Opt. Soc. Am. A 33, 913 (2016).

18. M. Graf, L. Emmenegger, and B. Tuzson, Opt. Lett. 43, 2434 (2018).

19. F. Wu, C. Li, W. Shi, J. Wei, and L. Deng, Spectrosc. Spect. Anal. 36, 1051 (2016).

20. Z. Yang, M. Zou, and L. Sun, Opt. Express 27, 32883 (2019).
21. Z. Yang, Y. Guo, X. Ming, and L. Sun, Sensors. 18, 2680 (2018).

22. M. Graf, H. Looser, L. Emmenegger, and B. Tuzson, Opt. Lett. 42, 3137 (2017). 